\title{
Walter Benjamins Umgang mit der Schrift
}

\author{
[Walter Benjamin on Writing] \\ http://dx.doi.org/10.1590/1982-88373757685
}

\section{Geors Otte}

\begin{abstract}
Walter Benjamin generally is considered as a "difficult" author, given that he does not work with previously defined and universally valid concepts, but lends a specific sense to existing concepts, such as Erlebnis, the individual, "modern" experience, and Erfahrung, the collective, pre-modern one. This, however, is only possible by the insertion of these "individual concepts" in certain contexts. The repetition of these concepts on the surface of the text, considered strange to each other, enter in an unexpected relationship, coming together, like in his theory of history, in a "dialectic image".
\end{abstract}

Key-Words: Walter Benjamin, Writing, Concept

Resumo: Walter Benjamin geralmente é considerado como um autor „difícil”, uma vez que não trabalha com conceitos anteriormente definidos e universalmente válidos, mas confere a conceitos existentes, tais como a moderna "vivência" individual ou a coletiva "experiência" prémoderna, um significado específico. Mas isso só é possível mediante a inserção desses "conceitos individuais" em determinados contextos. A repetição desses conceitos na superfície do texto faz com que contextos, tidos como estranhos entre si, entrem numa relação inesperada, encontrando-se, de maneira semelhante à teoria da história, numa "imagem dialética".

Palavras-chave: Walter Benjamin, Escrita, Conceito

Zusammenfassung: Walter Benjamin gilt allgemein als „schwieriger“ Autor, da er nicht mit vorab geklärten, universell gültigen Begriffen arbeitet, sondern vorhandenen Begriffen wie dem individuellen „Erlebnis“ in der Moderne oder der kollektiven „Erfahrung“ in der Prämoderne eine besondere Bedeutung verleiht. Dies ist jedoch nur dadurch möglich, dass er diese "Individualbegriffe“ in bestimmte Zusammenhänge einbettet. Die Wiederholung solcher Begriffe an der Textoberfläche bewirkt, dass einander als fremd vorgestellte Zusammenhänge in eine unerwartete Beziehung gebracht werden und, ähnlich wie in seiner Geschichtstheorie, zu einem „dialektischen Bild“" zusammenfinden.

Stichwörter: Walter Benjamin, Schrift, Begriff

\footnotetext{
${ }^{1}$ Universidade Federal de Minas Gerais (UFMG), Faculdade de Letras, Av. Pres. Antônio Carlos, 6627; Avenida Miguel Perrela, 31270-901, Belo Horizonte, MG. Email: georg@letras.ufmg.br

Pandaemonium, São Paulo, v. 18, n. 25, Jun. /2015, p. 57-68
} 
Otte, G. - Umgang mit der Schrift

Eine der Eigentümlichkeiten von Benjamins Texten ist die Schaffung eines eigenen Vokabulars und die daraus hervorgehende Schwierigkeit der Lektüre seiner Texte. Ein Buchtitel wie Benjamins Begriffe (OPITZ; WIZISLA 2011) ist im Grunde ein Widerspruch in sich, da man von Begriffen Allgemeingültigkeit erwartet und jede private Begrifflichkeit eine Zumutung an den Leser bedeutet. Der Aufbau einer individuellen Begrifflichkeit, logisch gesehen ein Unding, ist jedoch auch - oder gerade - im Bereich der Wissenschaften an der Tagesordnung. Selbst Naturwissenschaftler müssen den Gebrauch ihrer Begriffe klarstellen, um überhaupt innerhalb der wissenschaftlichen Gemeinschaft anerkannt $\mathrm{zu}$ werden. Umso mehr gilt dies für die hermeneutisch ausgerichteten Geisteswissenschaften, um auf Diltheys Unterscheidung zurückzugreifen, die zwar durch den linguistic turn (auch naturwissenschaftliche Beobachtungen sind sprachlich vermittelt) weitgehend überwunden, ihrer Tendenz nach aber immer noch aufschlussreich ist.

Die Lektüre wird dann zur Zumutung für den individuellen Leser und zu einem ethischen Problem für das „Denkkollektiv“ (FLECK 1980, 2010), wenn ein Autor glaubt, sich seine eigene Begriffswelt aufbauen zu können, bzw. den Gebrauch bestimmter Begriffe durch andere Autoren nicht berücksichtigen zu müssen. Um noch einmal auf Dilthey zurückzukommen: vergleicht man den Begriff des Erlebnisses bei Benjamin und Dilthey, so kommt man kaum über eine Herausarbeitung der Unterschiede hinaus. Benjamin erwähnt zwar gleich zu Beginn seines zweiten Baudelaire-Aufsatzes Diltheys Das Erlebnis und die Dichtung (GS I, 608²), sieht sich aber vermutlich mit seiner pauschalen Absage an die Lebensphilosophie der Aufgabe enthoben, seine wenige Seiten darauf folgende Erörterung des Erlebnis-Begriffes mit Dilthey in einen Zusammenhang zu bringen, geschweige denn ihn von ihm abzuleiten. Jede Ableitung als solche wird von Benjamin bereits seit seiner „Erkenntniskritischen Vorrede“ zum Trauerspiel-Buch verworfen und so sind auch einer begriffsgeschichtlichen Annäherung an Benjamins Begriffe enge Grenzen gesetzt, zumindest einer solchen. Weder die Logik noch die Begriffsgeschichte scheinen bei Benjamin viel ausrichten zu können.

Nicht sehr viel anders gestaltet sich Benjamins Verhältnis zu Freud, dessen Begriffe im erwähnten zweiten Baudelaire-Aufsatz zwar kurz diskutiert werden, danach jedoch nicht mehr zur Sprache zu kommen. Auch werden Freuds Begriffe und die seines Schülers Theodor Reik gleich bei ihrer Einführung im Text unmittelbar an

\footnotetext{
${ }^{2}$ Das Kürzel GS bezieht sich auf Benjamins Gesammelte Schriften (vgl. Bibliographie).
} 
Otte, G. - Umgang mit der Schrift

Prousts Unterscheidung zwischen der mémoire volontaire und mémoire involontaire angelehnt. Über die „Gedächtnisspur“, den „Reizschutz“ und die Durchbrechung dieses Reizschutzes im Trauma gelangt Benjamin bald zur „Chockabwehr“ bei Baudelaire und überhaupt machen in den Fußnoten Literaten wie Poe, Heine, Börne, Hoffmann und Valéry den Theoretikern bald den Rang streitig. Freud wird, wie gesagt, bald ganz aufgegeben, zumindest im Sinne von direkten Bezugnahmen. Wie Sigrid Weigel gezeigt hat, stehen solche expliziten Erwähnungen von Freud geradezu in einem umgekehrten Verhältnis zu den „Spuren Freudscher Denkfiguren“, deren große Bedeutung für Benjamin sich in seinem Gesamtwerk aufdecken lässt (WEIGEL 1997: $36)^{3}$

Wenn auch Marx, der noch im ersten Baudelaire-Aufsatz den Ton angab, in der zweiten Fassung nur noch eine Gastrolle spielt, so hat dies sicher mit Adornos Einspruch (BENJAMIN 1966: 782-789; vgl. a. AGAMBEN 2005) gegen einen Gebrauch marxistischer Begriffe zu tun, den dieser als allzu eigenwillig empfand und der dann auch die Veröffentlichung der ersten Fassung verhindert hat. Adornos Kritik hatte im Generalvorwurf einer fehlenden Vermittlung gegipfelt, sei es derjenigen zwischen den marxistischen Kategorien Über- und Unterbau, sei es der zwischen Theorie und Praxis, besonders aber im Verhältnis zwischen der ,staunende[n] Darstellung der bloßen Faktizität““ und dem „Gesamtprozeß“ überhaupt (BENJAMIN 1966: 786; Hervorh. Th. A.). Mit Freud scheint Benjamin in der zweiten Fassung einen neuen Anlauf genommen zu haben, begrifflich zwischen einem theoretischen Ansatz und der Realität einer Großstadt des 19. Jahrhunderts zu vermitteln und damit auch den Erwartungen Adornos zu entsprechen.

Es klingt allerdings wie Ironie, wenn Adorno in seiner begeisterten Reaktion auf die revidierte Fassung (GS I: 1130) ausgerechnet Benjamins Verwendung Freudscher Begriffe ausklammert und damit Benjamins Bemühen um Wissenschaftlichkeit ein weiteres Mal in Frage stellt. Nicht weniger ironisch mutet es an, wenn Adorno im gleichen Brief die „Theorie des Spielers“ als einen der Höhepunkte dieser Neufassung lobt, obwohl doch auch hier kaum von einer Theorie die Rede sein kann, da Benjamin sich darauf beschränkt, die Praxis zweier weit voneinander entfernter Lebensbereiche einander anzunähern. Denn Benjamin vergleicht den Glücksspieler mit dem Arbeiter an

\footnotetext{
${ }^{3} \mathrm{Im}$ übrigen sei an dieser Stelle betont, dass die in Sigrid Weigels Buch Entstellte Ähnlichkeit zusammengefassten Schriften grundlegend für jede Untersuchung von „Walter Benjamins theoretischer Schreibweise" - so der Untertitel - sind und auch für vorliegende Arbeit von größter Bedeutung ist.
} 
Otte, G. - Umgang mit der Schrift

der Maschine, ohne sich um den gesellschaftlichen „Gesamtprozeß“ zu kümmern, den Adorno mehrfach eingefordert hatte, und ohne die beobachteten Phänomene auf einen Begriff zu bringen. Der Vergleich zwischen dem Spieler und dem Arbeiter beruht allein auf der Gemeinsamkeit, dass beide den Chocks der modernen Stadt ausgesetzt seien. Der Spieler, der nicht arbeitet, wird dem tätigen Arbeiter mit Hilfe der Negativkategorie des Chocks unvermittelt an die Seite gestellt; ihre Gemeinsamkeiten haben nichts mit ihrer gesellschaftlichen Stellung, sondern allein mit ihren abrupten, von Chocks geprägten Bewegungsabläufen zu tun. Eine ähnliche Analogie hatte Benjamin bereits zu Beginn seiner ersten Fassung hergestellt, als er Napoleon III mit der Figur des Berufsverschwörers verglich (GS I: 613 f.) und diesen Vergleich mit der Unberechenbarkeit beider rechtfertigte. Natürlich konnte Benjamin hier auch nicht mit marxistischen Kategorien arbeiten, da sowohl der Spieler, als auch der anarchistische Berufsverschwörer als gesellschaftliche Randexistenzen für den Marxismus keine ernstzunehmenden Figuren sind.

Auch der zweite Baudelaire-Aufsatz leistet am Ende nicht die Arbeit am Begriff, die einer theoretischen Vermittlung zugrunde liegen sollte und Adornos Enthusiasmus hat wohl eher mit seinem von ihm selbst eingestandenen schlechten Gewissen angesichts seiner „nörgelnden Insistenz“ zu tun (GS I: 1130) als mit einer veränderten Haltung Benjamins im Umgang mit der Theorie; denn eine solche Veränderung hat bei ihm seit der „Erkenntniskritischen Vorrede“ trotz seiner zwischenzeitlichen Begegnung mit dem Marxismus nie stattgefunden. Das Problem besteht für Adorno auch nicht darin, dass Benjamin diesen Umgang mit der Theorie zeitlebens beibehalten hat, sondern in Benjamins sicherlich missglücktem Versuch, marxistisch zu argumentieren, bzw. die marxistischen Formen der Vermittlung zur Anwendung zu bringen.

Eine weitere Ironie besteht jedoch darin, dass Adorno bei seiner Kritik am ersten Baudelaire-Aufsatz auf Elemente der „Erkenntniskritischen Vorrede“ zu Benjamins Ursprung des deutschen Trauerspiels zurückgreift und damit sozusagen Benjamin gegen Benjamin ausspielt. In dieser Vorrede hatte Benjamin bereits erklärt, dass die Darstellung der Ideen auf einem immer, ,inbrünstigern Zurückgreifen auf die Phänomene [beruht], die niemals in Gefahr geraten, Gegenstände eines trüben Staunens zu bleiben, solange ihre Darstellung zugleich die der Ideen und darin erst ihr Einzelnes gerettet ist.“ (GS I: 225) Benjamin muss es wie eine Provokation empfunden haben, wenn Adorno ihm etwas vorwirft, was er, Benjamin, seinerzeit selbst abgelehnt hatte, 
Otte, G. - Umgang mit der Schrift

nämlich eine ,staunende Darstellung der bloßen Faktizität“. Adorno, der noch 1932 an der Frankfurter Universität ein Seminar zum Trauerspielbuch abgehalten hatte (BRODERSEN 1990: 212 f.), wusste sehr wohl, dass „Darstellung“ als solche für Benjamin weit mehr ist als eine positivistische Aufzählung von Fakten (vgl. a. OTTE 2013).

Es geht Benjamin eben nicht um „,bloße Faktizität“, sondern um eine, wie er in der Vorrede zum Trauerspielbuch sagt, ,gedrängte Positivität“ (GS I: 225), die mit Adornos Vorwurf des Positivismus nichts gemein hat, sondern die Voraussetzung für die Darstellung der Idee ist und mit deren Hilfe das „Bild der Welt in seiner Verkürzung“ (GS I: 228) gezeichnet wird. Es scheint aber eben auch diese gedrängte monadologische Verkürzung zu sein, die Adorno die nötige Vermittlung vermissen lässt, obwohl doch auch das Prinzip der Monade Anspruch auf Totalität erhebt. Aber diese auf sinnlicher Wahrnehmung der physischen Welt und damit ästhetisch fundierter Wahrnehmung von Analogien beruhende Totalität hat wenig mit Adornos hegelianischmarxistisch inspiriertem „Gesamtprozeß“ zu tun. Benjamin stellt den Prozesscharakter als solchen und damit auch die Rede vom „Gesamtprozeß“ in Frage, denn dieser scheint ihn nur in dem Maße zu interessieren, wie er auch von „Chocks“ unterbrochen werden kann.

Freilich stellt Benjamins Erkenntniskritik in der Vorrede den Leser z. T. vor erhebliche Verständnisschwierigkeiten und vielleicht ist es bezeichnend, wenn diese verstärkt auftreten, sobald es um die Vermittlung durch den Begriff geht:

Durch ihre Vermittlerrolle leihen die Begriffe den Phänomenen Anteil am Sein der Ideen. Und eben diese Vermittlerrolle macht sie tauglich $\mathrm{zu}$ der anderen, gleich ursprünglichen Aufgabe der Philosophie, zur Darstellung der Ideen. Indem die Rettung der Phänomene vermittels der Ideen sich vollzieht, vollzieht sich die Darstellung der Ideen im Mittel der Empirie. Denn nicht an sich selbst, sondern einzig und allein in einer Zuordnung dinglicher Elemente im Begriff stellen die Ideen sich dar. Und zwar tun sie es als deren Konfiguration. (GS I: 214)

So obskur die Vermittlerrolle des Begriffs bei Benjamin auch sein mag, sicher ist, dass er nicht auf begriffliche Abstraktion abzielt und vor allem auch nicht darauf, etwas von vorgängigen Begriffen abzuleiten oder etwas , auf den Begriff zu bringen'. Und wenn es um „die Darstellung der Ideen im Mittel der Empirie“ durch die Konfiguration dinglicher Elemente geht, fragt man sich als Leser, warum es überhaupt noch des Begriffs bedarf, da die Ideen durch die Phänomene unmittelbar - bzw. unvermittelt - 
Otte, G. - Umgang mit der Schrift

dargestellt werden sollen. Wenn die Idee andererseits „einem grundsätzlich anderen Bereiche als das von ihr Erfaßte“ angehört (GS I: 214), kann es im Grunde nicht mehr um Vermittlung, sondern nur noch um Darstellung gehen, wie er sie auch programmatisch im ersten Satz seiner „Vorrede“ postuliert: „Es ist dem philosophischen Schrifttum eigen, mit jeder Wendung von neuem vor der Frage der Darstellung zu stehen.“ (GS I: 207) Wie auch immer Benjamin sich die Vermittlerrolle der Begriffe denken mag, als gesichert kann gelten, dass er der Erkenntnis mit ihren deduktiven Ableitungen und induktiven Schlüssen die „intentionslose“ Kontemplation gegenüberstellt. Das atemlose oder atemberaubende Fortschreiten soll durch das meditative - eben kontemplative - Atemholen und damit durch Versenkung in den Gegenstand ersetzt werden:

Ausdauernd hebt das Denken stets von neuem an, umständlich geht es auf die Sache selbst zurück. Dies unablässige Atemholen ist die eigenste Daseinsform der Kontemplation. Denn indem sie den unterschiedlichen Sinnstufen bei der Betrachtung eines und desselben Gegenstandes folgt, empfängt sie den Antrieb ihres stets erneuten Einsetzens ebenso wie die Rechtfertigung ihrer intermittierenden Rhythmik. (GS I: 208)

Das ,philosophische Schrifttum“, das Benjamin auf diesen ersten Seiten der „Vorrede“ $\mathrm{zu}$ charakterisieren versucht und mit dem er konsequenterweise auch seine eigene, „performative Schreibweise“ meint (FINKELDE 2011), soll offenbar den horizontalen Fortgang in vertikalen Tiefgang verwandeln - eine Unterscheidung, die Erich Auerbach zur Bestimmung des biblischen Schrifttums im Gegensatz zur homerischen Epik benutzt hat (AUERBACH 1982: 19; 1987: 14). Um die unterschiedlichen, ,vertikalen“ Sinnstufen zu erfassen, kann das Denken und kann die Schrift, die dieses Denken darstellen soll, nicht einfach kontinuierlich weiterfließen, sondern muss sich gewissermaßen selbst unterbrechen, um so die vertikale Vertiefung zu ermöglichen. Freilich war es Benjamin nicht möglich, in seiner akademischen Arbeit solche Selbstunterbrechungen, wie sie in seinem Manuskript sichtbar sind, auch in der Druckversion graphisch zu reproduzieren (vgl. KLEINWORT 2011). Um seine eigene „intermittierende Rhythmik“ dennoch hervorzurufen, musste er das syntaktische Nacheinander durch das parataktische, unvermittelte Nebeneinander ersetzen.

Benjamin verweigert sich also - bis zu einem gewissen Grad - nicht nur der begrifflichen Vermittlung und damit der akademischen Diskussion seiner Zeit, die beispielsweise im Falle von Freud, aber auch der Phänomenologie nur in Form von Spuren in der Vorrede auszumachen ist, sondern auch einer syntaktischen Vermittlung 
Otte, G. - Umgang mit der Schrift

im Dienste einer logischen Synthese. Und es ist vielleicht die letzte große Ironie des Schicksals von Benjamins Schriften, dass Adorno nicht nur in seinem mit „Parataxis“ (ADORNO 1981: 447-491) betitelten Hölderlin-Vortrag von 1963, sondern vor allem in seinem einige Jahre zuvor verfassten Beitrag „Der Essay als Form“ (ADORNO 1981: 933; ADORNO 2003; 15-54) besagte Verweigerung als Qualitätsmerkmal der Gattung Essay hervorhebt und Benjamin posthum als deren „unerreichten Meister“ (ADORNO 1981: 20) präsentiert. Auch hier verweist Adorno auf die „Erkenntniskritische Vorrede“ und übernimmt aus dieser stillschweigend Benjamins „Begriffe“ und Wendungen wie diejenige vom „lückenlosen Deduktionszusammenhang“, dem der Essay sich verweigere, da er Totalität nur ,aufleuchten“ lasse (GS I: 213; ADORNO 1981: 24 f). Auch bei Adorno ist die Zahl der zu Benjamin führenden „Spuren“ weitaus größer als die der expliziten Quellenverweise.

Begriffe können - logisch gesehen - nicht plagiiert werden, da sie ein der Kommunikation dienendes Gemeingut sind. Anders sieht es bei Begriffen aus, die von bestimmten Autoren geschaffen wurden, bzw. von ihnen eine bestimmte Prägung erhalten haben und deshalb normalerweise durch Anführungszeichen, d.h. als Zitate gekennzeichnet werden. Unabhängig von der ethischen Frage der Nichtkennzeichnung solcher „Individualbegriffe“ lässt sich sagen, dass auch durch solche Wortzitate eine mehr oder weniger unterschwellige, in vielen Fällen wohl auch unbewusste Kommunikation mit dem betreffenden Autor stattfindet - eine Kommunikation nach Art der kommunizierenden Röhren, deren Zwischenverbindung nicht immer sichtbar gemacht wird. ${ }^{4}$ Der Leser seinerseits ist deshalb aufgerufen, diese z.T. untergründigen Zwischenverbindungen wieder herzustellen. Die von Benjamin mehrfach beschworene „Geistesgegenwart“ betrifft auch die Aufmerksamkeit des Lesers, die sich nicht so sehr auf das Verfolgen von Gedankengängen, sondern das Aufdecken der besagten Zwischenverbindungen aufgrund von Wortwiederholungen bezieht: „Ihre höchste Manifestation [der Geistesgegenwart] ist das Lesen, das in jedem Falle divinatorisch ist.“(GS V: 639)

Das Besondere am Phänomen der kommunizierenden Röhren ist bekanntlich, dass der Flüssigkeitsstand bei allen Unterschieden in Größe und Form der Röhren immer der gleiche ist. Und so kommt auch die Aufdeckung der Querverbindungen

\footnotetext{
${ }^{4}$ Sigrid Weigel (1997) benutzt im bereits erwähnten Werk die Metapher der kommunizierenden Röhren für ihren Vergleich zwischen Benjamin und Foucault.
}

Pandaemonium, São Paulo, v. 18, n. 25, Jun. /2015, p. 57-68 
zwischen einzelnen Texten häufig erst durch die materielle Identität zustande, d.h. dadurch, dass das gleiche Wort in seiner äußeren graphischen Form - sozusagen an der Oberfläche des Textes - wiederholt wird und als Pegel dient. Für den Leser von „darstellenden“ Texten besitzt das einzelne Wort somit das Potential einer Proustschen Madeleine, das ihn unwillkürlich - im Sinne der mémoire involontaire - an eine vorausgehende Verwendung desselben Wortes erinnert. Und genauso wie die Madeleine erst dadurch ihre Einmaligkeit erlangt, dass sie in Lindenblütentee getaucht wird, also durch ihre besondere Geschmackskombination, sind es auch gerade die seltenen Wortkombinationen - wie die des ,lückenlosen Deduktionszusammenhangs“ aufgrund ihrer Seltenheit besonders dazu geeignet sind, durch schlichte Wiederholung eine Brücke zur Vergangenheit zu schlagen bzw. die Vergangenheit zu „zitieren“, so wie die Mode in der 14. Geschichtsthese „eine vergangene Tracht zitiert“ (GS I: 701). Im Gegensatz zur Verwendung eines allgemeingültigen Begriffs ist die Wiederholung einer besonders ausgefallenen Wortkonstellation ein Zitat, auch wenn es als solches nicht gekennzeichnet wurde.

Paradoxerweise schafft die Wiederholung einander entfernter Phänomene einschließlich des Wortes als graphischem Phänomen - einen Zusammenhang, der nicht auf dem kontinuierlichen und dynamischen Fortschreiten eines Textes beruht, sondern auf seiner Unterbrechung. Ähnlich wie in der Madeleine-Episode das Leben des Protagonisten zum Stillstand kommt, erfährt auch die Lektüre des Textes durch das déjà-vu der Wortwiederholung eine mehr oder weniger bedeutende Zäsur in der Abfolge seiner Lektüre. Eine solche wird bekanntlich beim Zitieren von größeren Textpassagen auch graphisch im Schriftbild deutlich gemacht.

So könnte auch der Ausdruck des ,intermittierenden Rhythmus“, mit dem Benjamin in der „Erkenntniskritischen Vorrede“ die sich selbst unterbrechende Schreibweise charakterisiert, seinerseits eine der vielen Spuren sein, die von der auf den etwa zeitgleich verfassten Aufsatz zu Goethes Wahlverwandtschaften verweisen, wenn es nämlich um Hölderlins Begriff der ,gegenrhythmischen Unterbrechung“ (Hölderlin apud BENJAMIN; GS I: 181) und damit um die Idee des „Ausdruckslosen“ geht. Denn diese Unterbrechung

[...] ist nur eine andere Bezeichnung jener Cäsur, in der mit der Harmonie zugleich jeder Ausdruck sich legt, um einer innerhalb aller Kunstmittel ausdruckslosen Gewalt Raum zu geben. In der Tragödie als Verstummen des Helden, in der Hymne als Einspruch im Rhythmus vernehmbar. Ja, man könnte jenen Rhythmus nicht genauer 
Otte, G. - Umgang mit der Schrift

bezeichnen als mit der Aussage, daß etwas jenseits des Dichters der Dichtung ins Wort fällt. (GS I: 182)

Die ,ausdruckslose Gewalt“, die Benjamin zur Gewalt des Ausdruckslosen macht und als etwas andeutet, das ,jenseits des Dichters der Dichtung ins Wort fällt ${ }^{\star 65}$, mag allein durch seinen theologischen Hintergrund bereits positiv gewendet sein, welcher in diesem Text immer wieder zum Tragen kommt. Aber der negative Charakter der Unterbrechung durch das Ausdruckslose wird auch dadurch aufgehoben, dass das Ziel dieser Negation bereits etwas Negatives, nämlich eine ,falsche, irrende Totalität““ (GS I: 181) ist und dass sich diese doppelte Negation somit auch logisch in ein Positives wendet. Wie auch später in den Geschichtsthesen lokalisiert Benjamin zwar den Ursprung der Unterbrechung des Kontinuums im Messianischen; gleichzeitig kann er sie aber auch durchaus logisch begründen: „Wo eine Kette von Begebenheiten vor uns erscheint, da sieht er [der Engel der Geschichte] eine einzige Katastrophe [...]“ (GS I: 697; Hervorh. W.B.) So eindeutig der historische oder politische Bezug dieser Katastrophe in den Geschichtsthesen auch sein mag, so deutlich ist doch auch, dass hinter dem besonderen Totalitarismus des Faschismus eine allgemeine „falsche, irrende Totalität" steckt.

Und auch hinter Benjamins Kritik am Fortschritt steckt letzten Endes eine Kritik an jeder Form des „horizontalen“ Fortschreitens: „Wo eine Kette von Begebenheiten vor uns erscheint“ heißt auch so viel wie ,Die Kette der Begebenheiten ist Schein', so wie jedes Kontinuum Schein ist, dessen Sprengung Benjamin in den Thesen mehr als einmal einfordert und der auch im Aufsatz über die Wahlverwandtschaften durch die Intervention einer ,ausdruckslosen Gewalt“ zu Stillstand kommen soll. Das gewaltsame Ausdruckslose soll in beiden Texten eine falsche, durch fortschreitende Bewegung gekennzeichnete Kontinuität sprengen, um einer wahren, unterschwelligen Kontinuität Platz zu machen, die Benjamin im 20. Jahrhundert nur noch negativ als das Ausdruckslose oder, wie es in der zweiten Geschichtsthese heißt, als ,schwache messianische Kraft“" (GS I: 694; Hervorh. W.B.) bestimmen kann.

Wie sehr auch immer die Unterbrechung der Kontinuität auf einem theologischen Fundament stehen mag, sicher ist, dass Benjamin allein in der Unterbrechung der Kontinuität ein Wahrheitspotential sieht, das die Dynamik des Fortschreitens nicht nur

\footnotetext{
${ }^{5}$ Vgl. hierzu das Kapitel „Die Dichtung als Einbruchstelle“(WEIGEL 2008: 113-140)
}

Pandaemonium, São Paulo, v. 18, n. 25, Jun. /2015, p. 57-68 
Otte, G. - Umgang mit der Schrift

negiert, sondern auch zum Stillstand bringt: das Ausdruckslose ist nicht nur die „kritische Gewalt“, die den Fluss des dichterischen Ausdrucks unterbricht, sondern es versetzt ihn auch in Erstarrung:

Daher darf kein Kunstwerk gänzlich ungebannt lebendig scheinen ohne bloßer Schein zu werden und aufzuhören Kunstwerk zu sein. Das in ihm wogende Leben muß erstarrt und wie in einem Augenblick gebannt erscheinen. [...] Wie die Unterbrechung durch das gebietende Wort es vermag aus der Ausflucht eines Weibes die Wahrheit gerad da herauszuholen, wo sie unterbricht, so zwingt das Ausdruckslose die zitternde Harmonie einzuhalten und verewigt durch seinen Einspruch ihr Beben. (GS I: 181)

Zweifellos nimmt Benjamin in seinem relativ frühen Wahlverwandtschaften-Aufsatz die später artikulierte Dialektik im Stillstand vorweg, wo es ebenfalls die negativ konnotierte Unterbrechung ist, die eine oft als positiv empfundene, überraschende Verbindung herstellt, die gerade durch ihren meteorenhaften Einschlag in den Text auf andere Textstellen oder andere Texte verweist und eine unerwartete Beziehung herstellt. Wenn es in der 17. Geschichtsthese lapidar heißt: „Zum Denken gehört nicht nur die Bewegung der Gedanken sondern ebenso ihre Stillstellung“ (GS I: 702), so wird einmal mehr deutlich, dass es Benjamin in diesem letzten, von den Zeitumständen geprägten Text nicht nur um die „Kette der Begebenheiten“ geht, sondern dass er auch hier seinen Grundgedanken aus der „Erkenntnistheoretischen Vorrede“ wieder aufnimmt, wo die „Kunst des Absetzens im Gegensatz zur Kette der Deduktion“ (GS I: 212; Hervorh. G. O.) steht.

Es sei dahingestellt, ob solche Wortwiederholungen wie im Fall der „Kette“ Zufall sind oder nicht, ob Benjamin sich bewusst oder unbewusst zitiert oder einfach aus seinem persönlichen Glossar schöpft, das wie ein Palimpsest (vgl. JENNINGS 2011) plötzlich durchbricht. Es dürften jedoch kaum Zweifel daran bestehen, dass seine Geschichtsthesen das Zitat nicht nur explizit zum Prinzip der Geschichtsphilosophie erheben (14. These), sondern als einzelne Schrift sein Gesamtwerk „monadologisch“ kondensiert, bzw. ,als Monade kristallisiert“, wie es in der 17. These heißt, die ihrerseits die Gesamtheit der Thesen zusammenfasst. Der Fixierung von geschichtlicher Zeit im Bild der Geschichte entspricht die Fixierung - oder Kristallisierung - der Begriffe in der Schrift, die im Falle der Thesen auch im Schriftbild deutlich wird. Auch diese Vorgehensweise hatte Benjamin bereits philosophisch in der „Vorrede“ artikuliert: 
Otte, G. - Umgang mit der Schrift

Damit bestimmt die Tendenz aller philosophischen Begriffsbildung sich neu in dem alten Sinn: das Werden der Phänomene festzustellen in ihrem Sein. Denn der Seinsbegriff der philosophischen Wissenschaft ersättigt sich nicht am Phänomen, sondern erst an der Aufzehrung seiner Geschichte. (GS I, S. 228)

„Das Werden der Phänomene fest[zu]stellen“ heißt hier nicht, das Werden konstatieren, sondern „feststellen“ bedeutet hier so viel wie fixieren. Wie so oft verleiht Benjamin auch hier einem Begriff, der sich im Kommunikationsprozess verflüssigt hat, eine neue „Festigkeit“, indem er ihn auf die Sinnstufe der wörtlichen Bedeutung zurückführt. Die „Feststellung“ ist einmal mehr eine Spur, die nicht nur an der Oberfläche der Signifikanten über die „Stillstellung“ zur „Darstellung“ führt, sondern auf eine Schreibweise verweist, durch die der Schein des endlosen kontinuierlichen Werdens sich - wenn auch nur blitzhaft - als ein wahres Sein enthüllt. Neben einer gewissen Verfremdung durch die Rückführung auf den sensus litteralis ist es vor allem die Wiederholung desselben Wortes in unterschiedlichen Konstellationen, durch die Benjamins Begriffe nicht nur ihre verschiedenen Sinnstufen abtasten, sondern permanent auf Distanz zu ihrem standardsprachlichen Gebrauch gehen und diesen in Frage stellen.

„Einmal ausgearbeitete Begriffe sind ihm [Benjamin] nicht applikativ und klassifikatorisch zur Hand, sondern müssen am neuen Gegenstand neu entfaltet und ausgearbeitet werden.“ (LINDNER 2011: 51) Burkhardt Lindners Erläuterungen zum Allegoriebegriff machen deutlich, warum „Benjamins Begriffe“ dem Leser nach wie vor viel zumuten. Dies gilt auch für den Übersetzer, dem pedantischsten aller Leser, der Benjamins Wortwiederholungen auch in der Zielsprache einhalten müsste, damit die „Schlüsselwörter“ - heute würde man diese als „Hyperlinks“ bezeichnen - ihren Zitatcharakter beibehalten. Dass dies aus stilistischen Gründen nicht geschieht - der gute Stil verbietet Wortwiederholungen - ist fast schon tragisch zu nennen, denn die Ersetzung dieser Schlüsselwörter durch Synonyme macht die genannte Zitatfunktion zunichte. So wird ausgerechnet das Wort „Spur“ als „traço“, „rastro“, „vestígio“ und auch als „,marca“ ins Portugiesische übersetzt, d. h. in Wörter, die alle den mehr oder weniger gleichen Sinn haben und von daher auch die Herstellung von Bezügen erlauben mögen, aber gleichzeitig viele Spuren in Benjamins Texten verwischen. 


\section{Literaturverzeichnis}

Adorno, Theodor W. Der Essay als Form. In: Tiedemann, Rolf (ed.). Noten zur Literatur. Frankfurt/M., Suhrkamp, 1981, 9-33.

ADORNO, Theodor W. O ensaio como forma. In: Tiedemann, Rolf (ed.). Notas de literatura I. Übers. und Vorwort Jorge de Almeida. São Paulo, Editora 34, 2003, 15-54.

ADORNO, Theodor W. Parataxis. In: Tiedemann, Rolf (ed.). Noten zur Literatur. Frankfurt/M., Suhrkamp, 1981, 447-491.

AgAmBen, Giorgio. O príncipe e o sapo. O problema do método em Adorno e Benjamin. In: Infância e história. Destruição da experiência e origem da história. Trad. Henrique Burigo. Belo Horizonte, Editora UFMG, 2005, 129-149.

AuerBACH, Erich. Die Narbe des Odysseus. In: Mimesis - Dargestellte Wirklichkeit in der abendländischen Literatur. Berna, Munique, Francke, ${ }^{7} 1982,7-27$.

AuERBACH, Erich. A cicatriz de Ulisses. In: Mimesis - A representação da realidade na literatura ocidental. Trad. George B. Sperber. São Paulo, Perspectiva, ${ }^{2} 1987,1$ 1-20.

BENJAMIN, Walter. Gesammelte Schriften. Bd. I. Rolf Tiedemann, Gerhard Schweppenhäuser (eds.). Frankfurt/M., Suhrkamp, ${ }^{3} 1990$.

Benjamin, Walter. Briefe. Gershom Scholem, Theodor W. Adorno (eds.). 2 Bde. Frankfurt/M.: Suhrkamp, 1966.

Brodersen, Momme. Spinne im eigenen Netz. Walter Benjamin - Leben und Werk. BühlMoos, Elster-Verlag, 1990.

FINKELDE, Dominik. ,Ad hominem'. Karl Barths und Walter Benjamins performative Sprachtheorie. In: Weidner, Daniel; Weigel, Sigrid (eds.). Benjamin-Studien, Bd. 2. Fink, München, 2011, 111-127.

FLECK, Ludwik. Entstehung und Entwicklung einer wissenschaftlichen Tatsache. Einführung in die Lehre vom Denkstil und Denkkollektiv. Frankfurt, Suhrkamp, 1980.

FLECK, Ludwik. Gênese e desenvolvimento de um fato científico. Introdução à doutrina do pensamento e do coletivo de pensamento. Trad. Georg Otte, Mariana Camilo de Oliveira. Belo Horizonte, Fabrefactum, Belo Horizonte, 2010.

JEnnINGS, Michael W. Double Take - Palimpsestic Writing and Image-Character in Benjamin's Late Prose. In: Weidner, Daniel; Weigel, Sigrid (eds.). Benjamin-Studien, Bd. 2. Fink, München, 2011, 33-44.

KLEINwORT, Malte. Zur Desorientierung im Manuskript der Vorrede zu Benjamins Trauerspielbuch. In: Weidner, Daniel; Weigel, Sigrid (eds.). Benjamin-Studien, Bd. 2. Fink, München, 2011, 87-107.

LINDNER, Burkhardt. Allegorie. In: Opitz, Michael; Wizisla, Erdmut (eds.). Benjamins Begriffe. 2 Bde. Frankfurt, Suhrkamp, ${ }^{3} 2011$.

OPITZ, Michael; Wizisla, Erdmut (eds.). Benjamins Begriffe. 2 Bde. Frankfurt, Suhrkamp, ${ }^{3} 2011$.

OTTE, Georg. Entre a mediação e a exposição - sobre o idioleto de Walter Benjamin. In: Cadernos Benjaminianos, n. 7 (Homenagem a Jeanne Marie Gagnebin), 2013, 89-99. http://www.periodicos.letras.ufmg.br/index.php/cadernosbenjaminianos/article/view/60 47; Zugriff: 28.02.15.

WEIGEL, Sigrid. Kommunizierende Röhren: Michel Foucault und Walter Benjamin. In: Weigel, Sigrid, Entstellte Ähnlichkeit. Walter Benjamins theoretische Schreibweise. Frankfurt/M., Fischer, 1997, 189-212.

WeIGEL, Sigrid. Von der Topographie zur Schrift - Benjamins Gedächtniskonzept und die Bedeutung der Freudschen Psychoanalyse. In: Weigel, Sigrid, Entstellte Ähnlichkeit. Walter Benjamins theoretische Schreibweise. Frankfurt/M., Fischer, 1997, $27-51$. 